\title{
EL PLANEAMIENTO Y LA REHABILITACIÓN URBANA HACIA EL LOGRO DE LA SOSTENIBILIDAD DE LAS CIUDADES
}

\section{Autora:}

Marianela Cruz Cabrera'

${ }^{1}$ Facultad de Arquitectura, Artes y Diseño

Universidad Tecnológica Equinoccial

Correo electrónico: arqmarianelacruz@yahoo.es

Teléf: 0984520158

Quito, Ecuador

\section{REVISTA DE INVESTIGACIÓN CIENTIFICA}




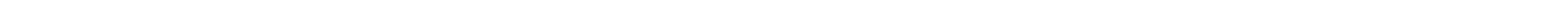




\section{RESUMEN}

En la actualidad las ciudades reflejan un crecimiento urbano imparable que conduce a la innegable necesidad de un marco metodológico desde el cual se puedan abordar los problemas a escala de las ciudades y encontrar soluciones viables y sostenibles. La investigación centra la atención en la planeación y la rehabilitación urbana como instrumentos esenciales del urbanismo hacia la meta de alcanzar la sostenibilidad de las ciudades, que permitirá responder a la interrogante de ¿cómo contribuir desde la escala del urbanismo al logro de ciudades eficientes, diversas y equitativas? Los métodos y técnicas de indagación y validación aplicados, combinan el trabajo en grupos de expertos, el análisis histórico-lógico de la información documental y el empleo de métodos estadísticos de procesamiento para investigaciones mixtas (cualitativas-cuantitativas) utilizando técnicas actualizadas de recogida y procesamiento de datos. Se explicita la importancia de la planeación, el planeamiento estratégico y la rehabilitación urbana hacia el desarrollo de una ciudad existente que posee identidad y significado en contraposición a un crecimiento incontrolado hacia límites insostenibles y poco manejables para los gobiernos locales.

Palabras claves: planeación urbana, renovación urbana

\section{ABSTRACT}

Today the urban reality reflects an unstoppable urban development that leads to the undeniable need for a methodological framework from which you can address the problem at the level of cities and find viable and sustainable solutions. The research focuses on urban planning and rehabilitation as essential instruments of urbanism toward the goal of achieving sustainability of cities, which will answer the question of how to contribute to achieving efficient, various and equitable cities from the scale of urbanism? The methods and techniques of inquiry and applied validation combine work in groups of experts, the historical and logical analysis of information and the use of statistical methods (qualitative-quantitative) using updated techniques for collecting and processing data. The importance of planning, strategic planning and urban regeneration to the development of an existing city with identity and meaning, not its uncontrolled development towards unsustainable and unwieldy limits are explicit.

Key words: urban planning, urban renewal 


\section{INTRODUCCIÓN}

Se reconoce que las ciudades necesitan nuevos instrumentos para gestionar un escenario cada vez más complejo y asegurar las dinámicas de crecimiento y el mantenimiento de los factores territoriales, sociales, económicos, culturales, políticos que las han convertido en centros dinámicos en sus respectivas sociedades. (Rey, 2010). Cada ciudad es responsable de descubrir sus potencialidades, resolver sus problemas internos, ser socialmente equitativa, fomentar la participación y gobernabilidad. Para ello el perfeccionamiento de su estructura física, resultado y sostén de todos los procesos que se desarrollan en su interior, es una de las acciones a realizar (Estambul, 1996). Se plantea que las ciudades son sus paisajes físicos y humanos. (Borja y Muxi, 2003) es contacto, regulación, intercambio y comunicación. La estructura urbana, la forma de producir la ciudad, el paisaje urbano, su monumentalidad, la movilidad, incluso el mercado, son aspectos secundarios o parciales en relación con aquello que es esencial de la ciudad, la interacción entre los ciudadanos y sus actividades e instituciones. (Rueda, 2008).

Urbanistas como Cerdá, Howard, Geddes, Le Corbusier y otros, han sido impulsores del primer objetivo de la ciudad, aumentar las probabilidades de contacto, intercambio y comunicación entre las personas, actividades, asociaciones e instituciones sin comprometer la calidad de vida urbana, (Rueda, 2008). La ciudad se reafirma como fenómeno histórico de alta complejidad en constante evolución, donde la calidad de vida se convierte en el parámetro más sustancial, por delante de los sistemas impositivos, las regulaciones urbanísticas o el costo del suelo. (García, 2004).

Es apreciable el consenso acerca de la influencia que ejerce el entorno en las personas, en su forma de comportamiento y cómo contribuye a la satisfacción de sus necesidades. Se reconoce que el nivel de autorrealización de un individuo a lo largo de su vida depende críticamente de la calidad del entorno en que se desenvuelve. (Boisier, 1999).

En la ciudad latinoamericana actual se observa el deterioro de su imagen, su fondo edificado y la infraestructura, agudizado por la imposibilidad de los gobiernos locales (unas veces económica y otras por falta de interés) para hacer frente a una situación acumulada por años. En el presente trabajo investigativo acerca de los procesos urbanos que atraviesa la ciudad se incorporan estas aristas para establecer la necesaria transformación en el marco físico-espacial de forma coherente y racional, a partir de dos instrumentos: la planeación y la rehabilitación urbana, en ambos casos bajo el enfoque del desarrollo sostenible de hacer una ciudad incluyente para todas las edades.

El concepto de sostenibilidad urbana (Conferencia N.U. sobre Medio ambiente y Desarrollo, Río, 1992) ha sido definido como: "Desarrollo que satisface las necesidades del presente sin condicionar las potencialidades de generaciones futuras de satisfacer los suyos." Sin embargo no debe constreñirse a los recursos naturales, sino extenderse a todas las capacidades humanas, en todas sus formas de capital: físico, humano, financiero, social y medioambiental.

La sostenibilidad urbana desde la visión de la planificación y la rehabilitación urbana, es considerada por la autora a partir de las posibilidades de definir lineamientos y acciones que benefician la calidad de vida de las personas, mejoran la respuesta de la ciudad, rescatan la ciudad tradicional, sus valores de identidad y significado para las generaciones actuales y futuras, de ahí que haya sido identificado como un principio a alcanzar en las propuestas urbanas para la obtención de ciudades más eficientes y diversas.

La sostenibilidad intenta perfilar nuevas estrategias en pro de la justicia, estrategias que no reclaman el enfrentamiento de capas sociales, sino a la solidaridad y la conciencia colectiva hacia los grupos menos favorecidos. (Hough, 1998). Es por ello que se considera de mucha importancia alcanzar el mejoramiento de la accesibilidad y la calidad de los espacios, favorecer los desplazamientos peatonales y el transporte público, generando espacios públicos seguros para el disfrute colectivo en una ciudad existente y que se transforma aceleradamente.

La planeación físico-espacial a través de una serie de mecanismos, acaba decidiendo los usos de suelo, analiza las condiciones de la población y el territorio, su modo de distribución y las condiciones paisajísticas. (López, 1999). De ahí que los procedimientos más utilizados actualmente tienden al logro de ciudades más compactas, que se basan en el aprovechamiento máximo del suelo urbano, solape de actividades, optimización del transporte urbano, recorridos mínimos limitando el uso del automóvil, mayor contacto entre las personas. Este modelo es proclamado como óptimo para el logro de un desarrollo urbano sostenible. (Rogers, 2000).

En el texto que se presenta se realizará unas reflexiones sobre la planeación y la rehabilitación urbana acompañada de ejemplos que argumentan la importancia de ser tomadas en cuenta e implementadas como formas viables de actuar en la ciudad, con el objetivo de alcanzar el desarrollo sostenible que el contexto urbano actual exige.

\section{MÉTODOS Y MATERIALES}

La ciudad latinoamericana en la actualidad refleja un crecimiento urbano imparable que conduce a la innegable necesidad de un marco metodológico desde el cual se puedan abordar dificultades identificados a escala de las ciudades y encontrar soluciones viables y sostenibles. Se aprecian debilidades en los 
procesos de planeación y rehabilitación urbana por lo que se hace cada vez más evidente la necesidad de continuar trabajando en el perfeccionamiento de los instrumentos metodológicos que orientan los procesos de planeación, diseño y gestión urbana, cuya práctica deberá orientarse hacia la búsqueda de la sostenibilidad de las ciudades.

El presente trabajo se basa en el análisis y compilación del estado del arte elaborado para la tesis doctoral "Adecuación del planeamiento físico espacial ante los cambios demográficos" donde se ratifica que el siglo XXI impone la adopción de nuevos caminos para la intervención urbana. Se debe estructurar la complejidad desde el enfoque sistémico y holístico en el urbanismo, como un requisito indispensable para enfrentar la problemática existente. También se resalta la rehabilitación urbana como actuación indispensable para la recuperación de la ciudad tradicional. La planeación y la rehabilitación urbana influyen notablemente en el trazado de adecuadas políticas urbanas en función de la satisfacción de las necesidades de la población y mejora de su calidad de vida. (Cruz, 2011).

El objeto de la investigación centra su atención en la planeación y la rehabilitación urbana como instrumentos esenciales hacia la meta de alcanzar la sostenibilidad de las ciudades, que permitirá responder la interrogante de ¿cómo contribuir desde la escala del urbanismo al logro de ciudades eficientes, diversas y equitativas?

El trabajo ha sido estructurado sobre la base de los métodos más generales del conocimiento científico, transita del análisis a la síntesis, utilizando el análisis histórico-lógico, análisissíntesis e inducción-deducción. Se explicita la necesidad de analizar con responsabilidad, la contribución de la planeación y la rehabilitación como instrumentos para la actuación a escala urbana, ante la existencia de puntos vulnerables en el modo de hacer ciudad en el contexto latinoamericano actual. Se ofrecen ejemplos que justifican la necesidad de retomar dichos instrumentos. Los métodos, técnicas de indagación y validación aplicados combinan el trabajo en grupos de expertos, las entrevistas y encuestas, el análisis de la información documental y el empleo de métodos estadísticos de procesamiento para investigaciones mixtas (cualitativas-cuantitativas) utilizando las técnicas actualizadas de recogida y procesamiento de datos.

Atendiendo a la complejidad de la problemática urbana se realizó una amplia discusión teórica basada en una bibliografía (AECI, FLACSO, CELADE, ILPES, CEPAL, ONU-Hábitat, PNUD). Como resultado se pudo apreciar que los temas abordados en el contexto urbanístico son atendidos en la generalidad de los países con especificidades en aquellos que se encuentran en vías de desarrollo. Se enfatiza en la necesidad de adoptar nuevos enfoques atemperados a la dinámica social y urbana, apoyados en las nuevas tecnologías y los avances científico técnico.

\section{LA PLANEACIÓN COMO INSTRUMENTO DEL URBANISMO}

La planeación urbana, espacial, social, económica e institucional permite ofrecer medios para organizar y regular el crecimiento de las ciudades, lo que constituye uno de los mayores retos que enfrenta el hombre en el siglo XXI. Un planeamiento que sea consciente del marco de incertidumbre y atento al carácter variable de los problemas urbanos así como de las tendencias, prioridades y modos de atender a esos problemas. (Alcalde y Arcusa, 1999).

Se necesita comprender la complejidad de las relaciones entre las diferentes actividades a escala urbana y la gente que la habita. Percibir los conflictos generados a lo largo de la historia, las posibilidades que provee el desarrollo, generados fundamentalmente por los rápidos cambios en la tecnología, las formas de intercambiar, comerciar y tendencias de consumo, evidenciadas tanto en el mundo desarrollado como en los países en vías de desarrollo, los cuales se constituyen en elementos esenciales a ser identificados y evaluados.

El Plan de Ordenamiento Urbano (POU) es un instrumento que permite determinar: los elementos estructurantes de la ciudad, según variables de análisis funcional, (residencial, industrial, servicios e infraestructuras entre otros); la protección de los recursos naturales y el medio ambiente, el patrimonio edificado, así como la morfología e imagen de la ciudad estableciendo un marco metodológico planificado, regulado y factible de controlar las acciones que vayan a acometerse.

Hasta mediados del siglo XX las formas de planear estaban en relación con una planeación tradicional, que se distinguió por su concepción de producto terminado, cerrado y de alta centralización. Posteriormente se definió un camino hacia la planeación de tipo estratégico y de acción, que aporta un producto continuo y flexible y tiene en cuenta el carácter siempre creciente de las necesidades de las personas.

En los procesos de planeación estratégica se prevé influenciar en el futuro de la ciudad utilizando el consenso y la coordinación de las acciones urbanas, económicas, políticas y sociales y no imponer un determinado tipo de estructuración y definición de usos de suelo como expresaría la planeación urbana tradicional. Esto marca la diferencia entre las dos formas de planear la ciudad.

La planeación estratégica aportaría elementos que llevan hacia la descentralización, el fortalecimiento de los vínculos con la gestión, la creación de capacidades para conducir los cambios y la flexibilidad con el objetivo de evaluar y asimilar las nuevas oportunidades, todo ello unido a una mayor participación institucional y ciudadana. Se integra de forma realista a los actores claves, mediante la identificación e inclusión temprana 
de estos en el proceso de análisis, síntesis y formulación de propuestas.

El arquitecto y urbanista José Miguel Fernández Güel en su texto, Planificación estratégica de ciudades: nuevos instrumentos y procesos, define:

La planificación estratégica en las ciudades es un proceso sistemático, creativo y participativo que sienta las bases de una actuación integrada a largo plazo, que define el modelo de desarrollo, que formula estrategias y cursos de acción para alcanzar dicho modelo, que establece un sistema continuo de toma de decisiones y que involucra a los agentes locales a lo largo de todo el proceso. (Fernández, J. M., 2006: 55).

La planeación estratégica como actividad puede alcanzar la integración de las diferentes visiones sectoriales existentes sobre una ciudad, permite desarrollar un enfoque pluridisciplinar y multisectorial que favorece cualquier análisis, por lo holístico de su alcance y por ende, favorece el logro de equidad, complejidad y sostenibilidad en un espacio físico determinado.

Otra característica importante de destacar es su visión de largo plazo y multidisciplinaria cuyo comportamiento es un proceso compartido y de consenso, que considera el entorno en su generalidad y en su estrecha relación con las personas que lo habitan, llevando el análisis hasta las relaciones entre el sistema de ciudades y el territorio que le es competente.

Como fenómeno complejo la ciudad responde al principio de recursividad organizacional establecido por Edgar Morín, donde los productos y los efectos son, al mismo tiempo, causa y efecto de aquello que lo produce, la ciudad es el reflejo de los individuos que la producen y a su vez, ésta después influye en el comportamiento de la población que reside y se mueve en ella. (Morín, 1998).

Como consecuencia, la planeación urbana permite alcanzar propuestas funcionales que contribuyen a la ordenación y planeación de ciudades más modernas para el presente y el futuro inmediato. La materialización de estas ideas se apoya en las fortalezas identificadas y determina una orientación hacia las acciones que tienen en cuenta la viabilidad y flexibilidad del alcance de las metas planteadas, los recursos que se requieren para su realización y sobre todo determina las responsabilidades según las competencias.

Como ejemplos significativos para ilustrar la aplicación de este tipo de planeación se han seleccionado, la transformación urbana de la ciudad de Curitiba en Brasil y Bogotá en Colombia y que a continuación se exponen de forma sintetizada y con énfasis en aquellos aspectos que han sido considerados de mayor relevancia.

\section{LA PLANIFICACIÓN DE LA CIUDAD HISTÓRICA DE CURITIBA, BRASIL}

Curitiba, ciudad de la región oriental del Paraná al sur del Brasil, cuenta con 1,7 millones de habitantes. El crecimiento urbano desde la última década del siglo XX hasta la actualidad se ha caracterizado por la ocupación de los espacios vacíos e intersticiales de la trama existente y una ocupación de áreas periféricas por funciones residenciales. Esta ciudad se propuso hacer cambios sustantivos en su configuración urbana en función de mejorar su medio ambiente natural y construido y hacia ese fin encaminó su planeación.

El cambio de la ciudad no se logró mediante grandes megaproyectos, sino debido a la ejecución de cientos de pequeños proyectos articulados, de bajo presupuesto pero de gran impacto social. Como plantea Jaime Lerner: La ciudad es una estructura de vida y de trabajo juntos. La ciudad es una integración de funciones. Cuanto más se integran las funciones urbanas, más humana quedará la ciudad, (Lerner, 2003).

Uno de los elementos innovadores del Planeamiento Estratégico de la Ciudad de Curitiba está constituido, por su atención dirigida al mejoramiento de la movilidad urbana y al rescate y preservación del medio ambiente con especial énfasis en el tratamiento, ampliación y mejoramiento de los espacios verdes dentro de la ciudad en articulación con los espacios públicos como generadores de la interacción social, todo ello en estrecha relación con los usos de suelo.

El plan otorgó especial atención a las áreas verdes, los espacios públicos, la movilidad urbana, la prioridad dada a los sistemas de transportes el cual fue analizado de forma tal que se interconectaran los diferentes medios con todas las zonas de la ciudad. El proceso de planificación muestra un enfoque sostenible al concebir la planificación desde tres aristas fundamentales:

“... el sistema vial, el uso del suelo y el transporte público. A partir de la cual la ciudad construyó su desarrollo económico, social y ambiental. La experiencia de la movilidad de Curitiba no se puede medir sólo por el análisis de su sistema de transporte público, que aún utiliza actualmente. Este sistema forma parte de un proceso mucho más complejo que incluye su implantación concomitante e integrada al uso del suelo y el sistema vial. La implantación y modernización permanente de la Red de Transporte Integrado (RIT), utilizando los autobuses articulados y bi-articulados en diferentes líneas de servicio así como los de interbarrios, línea directa, y la tarifa única e integrada, dio a la ciudad la capacidad de moverse en cualquier dirección, (OCO, Curitiba, 2011).

También se distingue un proceso de planeación urbana marcado por el concepto de accesibilidad de los servicios públicos y la

\section{TsaFiquilewax}


participación ciudadana, surgiendo las llamadas Calles de Ciudadanía, reuniendo en un mismo espacio físico los servicios públicos esenciales, áreas deportivas, culturales, convirtiéndose en atractivos y funcionales puntos de encuentro de las personas.

Cabe destacar de esta experiencia, la obtención de resultados que hasta hoy día se constituyen en referentes de la planificación urbana hacia la sostenibilidad, entre los que se pueden expresar como más significativos los siguientes: Jerarquizar los espacios verdes y públicos como importantes áreas que favorecen los vínculos e interacciones de carácter social, priorizar el espacio vial para el transporte público frente al transporte individual; mejorar y ampliar la integración del transporte público y la integración metropolitana; promover la accesibilidad a través de una red integrada de vías, ciclovías y calles peatonales de forma segura y cómoda para todas las personas, haciendo énfasis en aquellos con movilidad limitada. Como oportunidad urbanística el uso del Sistema de Transporte fue utilizado como una herramienta para inducir la ocupación urbana ordenada, cuando se trabaja en conjunto con el sistema vial y el uso del suelo. (OCO, Curitiba, 2011)

\section{EL PLAN ESTRATÉGICO DE BOGOTÁ 1994 - 2007 “LA CONSTRUCCIÓN DE UN MODELO"}

Este es un plan que llama la atención por su implicación en la visibilidad internacional que alcanzó la ciudad, basado en las soluciones planteadas a los problemas urbanos identificados. Se destaca la continuidad que ha tenido la planeación urbanística y el enfoque hacia una cultura ciudadana, el tratamiento de los espacios públicos y muy especialmente el tema del transporte. La temática del transporte no sólo intervino en el sistema de movilidad sino que también trajo aparejada una transformación urbanística que contribuyó a ordenar la ciudad a partir del proyecto del Transmilenio, que revolucionó la imagen y el paisaje de la ciudad conjuntamente con las ventajas de la accesibilidad a diferentes áreas urbanas.

El abordaje de los espacios públicos ha sido un tema digno de destacar, pues ha definido como prioridad alcanzar mejoras ostensibles en la calidad de vida de sus habitantes y de aquellos que la visitan, implementando un sistema de parques, plazas, alamedas y ciclo-vías que han modificado en gran medida el uso y disfrute del tiempo libre de los habitantes de esta hermosa ciudad.

En el Informe de Desarrollo Humano presentado a finales de 2007, se señala que los indicadores sociales mostraron un mejoramiento en las condiciones de vida de las personas, una disminución histórica de la pobreza en la ciudad al bajar de 38,3 por ciento en 2002 a 23,8 por ciento en 2006. (Duque Franco, 2008). Sin embargo, Bogotá continúa siendo una ciudad donde no se ha podido eliminar la segregación urbana y social, por lo que la continuidad del planeamiento necesita de la participación ciudadana e institucional ya que la complejidad urbana así lo requiere.

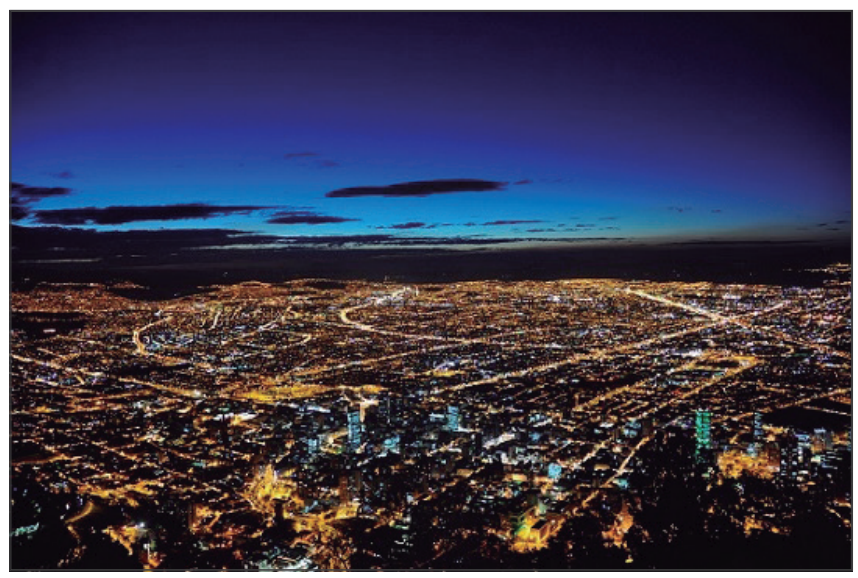

Foto 2. Ciudad de Bogotá un proyecto de ciudad y un referente para si misma. Fuente: Internet

Resumiendo los ejemplos expuestos (Planes Estratégicos de Curitiba y Bogotá) es posible decir que tienen como punto en común el hecho de que fueron planificados e implementados y los logros alcanzados en el mejoramiento de las deficiencias a escala urbana, tienen su basamento en la existencia de una planeación urbana con visión de futuro. Dicha planeación ha logrado ordenar mediante la definición de prioridades para las propuestas de solución y acciones a ejecutar compatibilizadas con los recursos financieros, humanos y materiales disponibles.

La planeación ha sido centrada en el mejoramiento de la calidad de vida de los habitantes y una amplia atención a la preservación y mejoramiento del medio ambiente, especial atención otorgada a los temas de movilidad urbana y accesibilidad, en relación estrecha con los usos de suelo, el tratamiento de los espacios públicos y una clara línea de apropiación y cuidado de los recursos que se disponen.

\section{LA REHABILITACIÓN URBANA EN LA PRESERVACIÓN DE LA CIUDAD}

En la segunda parte de este trabajo se considera importante llamar la atención sobre los procesos de Rehabilitación Urbana, considerando su importante aporte en el rescate de la ciudad tradicional. Esta forma de intervención conduce de forma innegable al logro de la sostenibilidad urbana y se sustenta a partir del rescate y aprovechamiento de áreas urbanas de gran valor y con una infraestructura consolidada. 
Se aprecia un crecimiento sin precedentes en las ciudades de todo el mundo en desarrollo, planteando un desafío significativo en América Latina y el Caribe, que experimentó un crecimiento explosivo hacia la rápida expansión de la periferia urbana. (Rojas, 1998) y cierto abandono de la ciudad tradicional, sin embargo hubo una toma de conciencia encaminada a su rescate. El arquitecto ecuatoriano Fernando Carrión ha descrito a las ciudades latinoamericanas planteando como características más significativas la Fragmentación: mosaico de espacios inconexos y desarticulados. Segmentación: diferencia entre ricos y pobres, cada uno posee sus zonas. Difusión: urbanizaciones periféricas con bajas densidades, centralidades débiles y espacios discontinuos. Inseguridad: la violencia impacta la ciudad y Privatización: discusión entre lo público y lo privado afectando el funcionamiento de la ciudad y a sus habitantes. (Carrión, 2004). Se ratifica la necesidad de abordar la ciudad tradicional y tejerla con la nueva ciudad en desarrollo.

Sería interesante realizar un recorrido sobre aquellos momentos históricos que han marcado de forma significativa la importancia que posee el rescate y recuperación de la ciudad tradicional. La Carta de Atenas de 1931, planteó la necesidad de que la utilización de los monumentos garantizase la continuidad de su existencia, planteándose acciones de restauración que no perjudicaran los estilos de ninguna época.

A partir de los años 60 ocurrieron procesos que condujeron a la marginalización de los centros históricos y en los casos más dramáticos se llegó a su propia desaparición. Se observó la sustitución de edificios de alto valor arquitectónico por construcciones en altura que respondían a procesos de especulación del suelo y que fue borrando la imagen de ciudad tradicional y los valores de identidad de la ciudad.

La Carta de Venecia en 1964, expresó que la noción de patrimonio histórico no solo comprende la obra aislada sino que incluye también al sitio urbano o rural que dé testimonio de una civilización particular. Mediante este documento se consagró la extinción del concepto de patrimonio de los edificios al ambiente urbano y quedó claro que conservar la ciudad tradicional por sí sola no garantizaba el futuro y que era necesario vincular el plan urbano de la ciudad con un plan especial para el Centro Histórico, que permitiera el rescate de la imagen en su totalidad no aisladamente, además de desarrollar la capacidad de ser entendida por su estructura armónica y coherente llena de simbolismo e identidad.

Con posterioridad las Normas de Quito en el año 1967 sentaron un sólido precedente al plantear que la idea del espacio es inseparable del concepto de monumento, otorgando a estas áreas el valor añadido de la presencia de la población como parte inseparable del patrimonio. Preservar la memoria histórica y el sentido de identidad unido al mejoramiento de las condiciones de vida de la población.
Ya entrado los años 70 se comienzan a delimitar las zonas urbanas asociadas al núcleo fundacional y son reconocidas como zonas especialmente valiosas, y por lo tanto requeridas de un tratamiento especial de salvaguarda. Comienza a desarrollarse una forma de intervenir a escala urbana que devuelve sus valores a estas áreas de la ciudad.

La ocurrencia de grandes desastres de origen natural como los terremotos ocurridos en Ciudad de México, 1985 y en la ciudad de Quito, 1987, provocó una reacción encaminada a tomar acciones hacia la conservación de los centros históricos esenciales. En 1990 ocurre un cambio radical en la forma de actuar en los centros históricos en Latinoamérica, otorgando especial atención a las acciones de rehabilitación a escala urbana.

Las ciudades sufren un innegable deterioro con el paso del tiempo, debido a la falta de mantenimiento que se ha venido acumulando, (tanto en las edificaciones como en sus infraestructuras), unido a un incremento no planificado de población. Debido a esto los gobiernos locales buscan alternativas para implementar programas de rehabilitación urbana que se puedan llevar a vías de hecho y que a su vez sean sostenibles, apoyados en procesos de valorización de la cultura, puesta en valor y rescate de la identidad, las tradiciones y la salvaguarda del patrimonio edificado.

La rehabilitación urbana se puede describir como un proceso de revitalización y refuncionalización del contexto urbano. Es un tipo de intervención que abarca el entorno físico-ambiental y el conjunto de actividades sociales, culturales y económicas que se manifiestan en el entorno de las ciudades. Su objetivo se encamina al mejoramiento de las condiciones de vida de la población y la mejora de la calidad del entorno construido, teniendo en cuenta el contexto existente en el momento de la actuación cuya meta será recuperar y actualizar funciones que ya no existen o han sido dañadas en la ciudad.

Las acciones de rehabilitación resultan complejas, y deberán ser abordadas como procesos de transformación lento y de forma programada, nunca de intervenciones violentas, sin planificación previa. Plantearse la intervención de forma aislada o parcial, buscando únicamente resultados inmediatos, acarrean consecuencias imprevisibles y en muchos casos pérdidas patrimoniales irrecuperables.

\section{La Carta de Lisboa plantea que la Rehabilitación Urbana es:}

Estrategia de gestión urbana para recalificar una ciudad existente a través de múltiples intervenciones destinadas a valorizar su potencial social, económico y funcional a fin de mejorar la calidad de vida de las poblaciones residentes. Ello exige el mejoramiento de las condiciones físicas del parque construido a través de su rehabilitación, instalando

\section{Tsafiquiew=}


equipamientos, infraestructuras y espacios públicos y conservando así la identidad y las características del área de ciudad considerada. (Carta de Lisboa, 1995)

En la actualidad la Rehabilitación Urbana ha tomado mucha fuerza, por ser considerada sinónimo de sostenibilidad en el manejo del espacio urbana, debido a que promueve la reutilización de un lugar consolidado en el tiempo, que tiene historia, identidad, simbolismo y significado.

Algunos de los riesgos más comunes a los que se enfrentan los centros urbanos en proceso de rehabilitación son en primer lugar, los desequilibrios en la función y ocupación del suelo que han sido generados por un incremento excesivo del turismo, que reduce y altera las necesidades del lugar y la gente que lo habita como barrio normal de una ciudad. También son modificadas las funciones residenciales, que en la mayoría de los casos tienden a la ghettificación de las áreas centrales y en el peor de los casos al despoblamiento y abandono por parte de la población.

Constituyen además elementos de riesgo las actuaciones urbanísticas y arquitectónicas o cambios de usos sin la debida planificación, abandonando las necesidades sociales de la población residente en estas zonas.

Atendiendo a la problemática antes descrita se han identificado algunos ejemplos que pueden demostrar la pertinencia de la renovación urbana mediante procesos de rehabilitación, al intervenir en la identificación y mitigación de los riesgos que presentan estas importantes y significativas áreas de la ciudad.

\section{PLAN DE REHABILITACIÓN URBANA DE BOLONIA, ITALIA (1969-1972)}

Bolonia, posee una estructura urbana medieval. Constituye una importante referencia para las generaciones de urbanistas, en la recuperación de centros históricos, destacando en su actuación conceptos como la triple intervención: Usos -HabitantesTipologías edificatorias.

La renovación del centro histórico de Bolonia, dirigida por Cervellati, tuvo como objetivo fundamental la lucha contra su degradación cultural y social, el desalojo de la población residente hacia la periferia y la sustitución de usos residenciales por otros más rentables. Esta rehabilitación realizada en el año 1970 fue la primera propuesta conservacionista desde el inicio del Movimiento Moderno.

A lo largo del tiempo esta transformación urbana se ha convertido en el "paradigma" de lo que debe entenderse hoy por "Recuperación de la ciudad histórica" conservando, tanto los edificios, como la población residente. Su objetivo urbanístico fue la preservación de la funcionalidad de la ciudad histórica, mediante su incorporación a la estructura urbana de la ciudad, como una parte diferenciada y cualificada a través de su propia identidad. Estar articulada a la ciudad y no ser un área relegada e indiferente.

La intervención en esta ciudad condujo a la denominada forma de conservación estructural, en la que las acciones se encaminaron al logro de la protección del Centro Histórico, la integración al patrimonio edificado de funciones apoyadas en equipamientos sociales. Se racionalizó el uso del transporte que favoreció la preservación de sus valores arquitectónicos y patrimoniales beneficiando el tránsito peatonal.

El Plan modificó la visión más romántica de Centro Histórico hacia una imagen de historia e identidad estrechamente vinculada a una morfología de la estructura urbana existente y consolidada. Se propuso la conservación de la forma de la ciudad relacionando los tejidos a conservar y los cambios necesarios de restauración, rehabilitación, reconstrucción. Demostró además que es posible el uso renovado de la función residencial en contextos comprometidos como es la ciudad histórica.

\section{REHABILITACIÓN DE LA CIUDAD HISTÓRICA DE VITORIA, ESPAÑA}

La ciudad histórica de Vitoria, se constituye en el cuerpo fundacional de la ciudad y comprende la estructura medieval que significó los límites de la misma hasta el siglo XVIII y que con el paso del tiempo se había convertido en un área descualificada y rechazada.

La rehabilitación del casco histórico en el año 1982 resolvió el principal foco de marginalidad existente en la ciudad. Constituyéndose en un modelo de desarrollo urbano de calidad, porque supo mantener un crecimiento urbano de mayor calidad, respetuoso con el patrimonio, la habitabilidad y el entorno.

A partir de 1982 la rehabilitación integral de esa zona de la ciudad aborda situaciones existentes en la trama urbana y el tejido social, entrando a resolver problemas de desorden, falta de higiene y servicios, congestión del tráfico, insuficientes estacionamientos y un alto deterioro del fondo habitacional, que provocaban serias amenazas contra una ciudad cuya calidad de vida poseía ciertos estándares y sin una intervención se corría el riesgo de la ghettificación de las áreas centrales de la ciudad.

La planeación urbanística conjuntamente con la rehabilitación de las áreas centrales de la ciudad de Vitoria, incrementó la trama verde del conjunto urbano y facilitó la proximidad entre el centro y el resto de las áreas, impulsando variadas políticas de movilidad que buscaron favorecer al peatón y los medios alternativos de transporte. Conjuntamente 
fueron implementados planes de urbanización, iluminación, saneamiento y recuperación de aquellos edificios que se distinguían por sus valores arquitectónicos, urbanísticos y ambientales.

\section{REHABILITACIÓN DE LA CIUDAD HISTÓRICA DE SANTIAGO DE COMPOSTELA, ESPAÑA}

En el año 1985 la ciudad fue declarada Patrimonio de la Humanidad por la UNESCO. Se elaboró un Plan de Rehabilitación de la Ciudad Histórica, que pretendía recuperar y proteger las funciones urbanas del centro histórico y la defensa y reconstrucción de los valores urbanos y arquitectónicos de la ciudad.

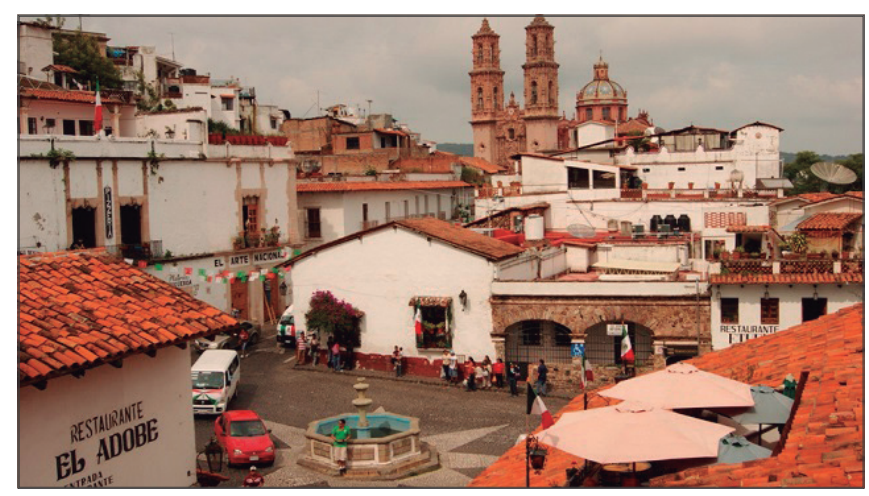

Foto 3. La conservación de la ciudad tradicional reafirma la identidad y significado del espacio urbano.

Fuente: Internet

Para ello se trazó tres objetivos prioritarios de actuación en el centro histórico: darle uso residencial a la zona, convertirla en un lugar de encuentro y crear corredores verdes a su alrededor. Se convirtió en un referente de proyecto urbano, siendo la conservación y puesta en valor de su patrimonio histórico el motor de la transformación de la ciudad.

Es importante de este plan de rehabilitación resaltar dos aspectos medulares: el primero su carácter educativo, que impulsó la formación de trabajadores de la construcción en los temas de rehabilitación de entornos patrimoniales y en segundo lugar el rol participativo, que propició que la población comprendiera y apoyara el proceso.

\section{PLAN MAESTRO PARA LA REVITALIZACIÓN INTEGRAL DE LA HABANA VIEJA}

La rehabilitación urbana para la revitalización integral de la Habana Vieja, (1994), se realizó bajo la dirección de un equipo multidisciplinario que llevaría a cabo la importante labor de rescatar los valores patrimoniales de la zona, conjuntamente con la transformación social. La rehabilitación del Centro Histórico de La Habana se ejecutó siguiendo pautas como: la compatibilización de los valores culturales con el desarrollo socioeconómico, conservando su carácter residencial y garantizando la sostenibilidad.

Con esta intervención se logró generar, dentro de su perímetro urbano, los recursos necesarios para la recuperación, mediante la articulación de proyectos sociales y otros que produzcan fondos económicos para la reinversión sistemática en planes a corto, mediano y largo plazo. El desarrollo de un amplio programa social, que apoya el concepto de un centro histórico vivo, hizo presentes en la zona todos los servicios asociados al hábitat y el mejoramiento de la calidad de vida de las personas unido al entorno en que viven.

En este ejemplo se evidencia un proceso de intervención que se clasifica como abierto y continuo, basado en la prospectiva y el planeamiento estratégico planteando soluciones concretas para la resolución de los conflictos.

Los ejemplos antes expuestos permiten corroborar que la rehabilitación urbana es sin lugar a dudas una importante forma de recuperar la ciudad y hacerla más eficiente y competitiva y se demuestra que, es posible evitar la degradación y el aspecto marginal de las áreas centrales de la ciudad, gestionar una movilidad menos agresiva y más sostenible en estas áreas unido a una recuperación de valores que representan identidad y cultura de cada país. También se pueden generar los recursos necesarios que contribuyan a mantener los logros alcanzados en función de la ciudad tradicional recuperada.

Se evidencia la existencia de problemas comunes entre ciudades, independientemente de su desarrollo o sistemas socioeconómicos diferentes, por lo que resulta conveniente aplicar instrumentos que viabilicen los procesos de análisis y formulación de propuestas para mejorar los asentamientos urbanos que ya existen y hacerlos más inclusivos y agradables a la vida social."(Platter Ziberk y Duany, 2010). Se puede eliminar la marginalidad, el desarrollo periférico incontrolado y el deterioro del fondo edificado mediante el uso de la planeación y la rehabilitación urbana en aras de alcanzar el desarrollo sostenible de las ciudades.

\section{Tsafiquilewas}




\section{CONCLUSIONES}

- Mediante la planeación y rehabilitación urbana se puede alcanzar la consolidación de la ciudad existente, unida a la recuperación de la ciudad tradicional y limitar el crecimiento incontrolado hacia límites insostenibles y poco manejables por los gobiernos locales.

- Es posible el mejoramiento de las deficiencias a escala urbana, cuando su basamento parte de la existencia de una planeación urbana con visión de futuro y la definición de prioridades para las acciones a ejecutar es compatibilizada con los recursos financieros, humanos y materiales disponibles.

- La planeación urbana debe poner especial atención a los temas de movilidad urbana y accesibilidad, en articulación con los usos de suelo y el tratamiento de los espacios públicos con una clara línea de apropiación y preservación de los recursos que se disponen.

- La rehabilitación urbana posee un carácter educativo, hacia la formación de trabajadores de la construcción en los temas de rehabilitación de entornos patrimoniales de alto compromiso y potencia el rol participativo de la población residente que permite una mayor comprensión y apoyo a la intervención. Demuestra que es posible recuperar la ciudad tradicional, reflejo indiscutible de la historia e identidad de un lugar, respetando las formas urbanas que le dieron origen (calles, manzanas, plazas).

- La rehabilitación urbana es una forma de intervenir en la ciudad que favorece la generación de recursos necesarios para la recuperación urbana, siempre y cuando logre articular proyectos sociales y otros que produzcan fondos económicos para la reinversión sistemática en planes a corto, mediano y largo plazo.

\section{REFERENCIAS BIBLIOGRÁFICAS}

1. Alcalde Fernández Mercedes, Arcusa Moragrena Gema. (1999) La necesidad de Planeamiento Urbano en la Construcción de la ciudad. Edita: Instituto Juan de Herrera. Madrid. España. ISSN: 1578-097X

2. Boisier, Sergio. (1999). Teorías y Metáforas sobre el desarrollo territorial. Editorial de la ONU, Santiago de Chile p25

3. Borja, Jordi y Zaida Muxí. (2003). El gobierno del territorio de las ciudades latinoamericanas.

4. Borja, Jordi; Muxí, Zaida. (2003). Espacio Público: ciudad y ciudadanía. Ed. Electa, Barcelona, España.

5. Carrión, Fernando. (2004). Espacio Público: Punto de Partida para la alteridad. Ponencia en el Libro: Ciudad e
Inclusión por el Derecho a la Ciudad. Editorial Gente Nueva, La Habana, Cuba, p65

6. Cruz Cabrera, M. (2011). Adecuación del planeamiento físico espacial ante los cambios demográficos en ciudades principales. Tesis doctoral Universidad Central - Marta Abreu\| de Las Villas, Santa Clara, Cuba.

7. Duque Franco, Isabel. (2008). Planeamiento urbano en Bogotá 1994-2007. La Construcción de un Modelo. X Coloquio Internacional de Geocrítica, Universidad de Barcelona.

8. Estambul, Declaración sobre los Asentamientos Humanos. (1996) Capítulo II Metas y Principios, Artículo 30, Turquía.

9. Fernández Güell, José Miguel (2006). Planificación Estratégica de las ciudades. Nuevos Instrumentos y procesos. Barcelona, editorial Reverté, (Nueva edición revisada y aumentada). ISBN 10:84-291-2110-2

10. García Vázquez, Carlos. (2004). Ciudad Hojaldre. Visiones urbanas del Siglo XXI editorial Gustavo Gili, SA, Barcelona, España, p-58

11. Hough, Michael. (1998). Naturaleza y ciudad. Planificación urbana y procesos ecológicos. versión castellana, editorial Gustavo Gili, SA, Barcelona, España p-94

12. Lerner, J. (2003). Acupuntura Urbana. Rio de Janeiro: Record, ps. 39 e 40.

13. López Candeira, José A. (1999). Diseño Urbano. Teoría y Práctica." Editorial Munilla- Lería Madrid, España.

14. Morín, Edgar. (1998). Introducción al pensamiento complejo. Gedisa, segunda reimpresión, Barcelona, España.

15. Oficina de Coordinación y Orientación (OCO), (2011). Sistematización de la experiencia, Plan de Movilidad Urbana Curitiba, Brasil.

16. Rey Rodríguez, Georgina. (2010). Instrumentos innovadores para la intervención urbanística en áreas centrales urbanas. Tesis Doctoral, La Habana.

17. Plater Zyberk y Duany. (2010). Smart Code Un código inteligente de Diseño Urbano V9.2. Ediciones Unión, Colección de Arquitectura y Urbanismo, La Habana, Cuba

18. Rogers, Richard. (2000). Ciudades para un pequeño planeta. Editorial Gustavo Gili, SA, Barcelona, España.

19. Rojas, Eduardo. (1998). Revitalización urbana, La ciudad en el siglo XXI. Quito, Ecuador.

20. Rueda Salvador. (2008). La ciudad compacta y diversa frente a la conurbación difusa. Dirección General de la Vivienda, la Arquitectura y el Urbanismo del Ministerio de Fomento de España. 
